\title{
Papers
}

\section{Perceptions of stroke in the general public and patients with stroke: a qualitative study}

Sung Sug Yoon, Julie Byles

\begin{abstract}
Objectives To gain insight into people's thoughts on stroke and to inform the development of educational strategies in the community.

Design Focus group discussions: two groups of people who had a stroke and their carers, and two groups of members of the general public.

Setting New South Wales, Australia.

Participants 35 people participated: 11 from the general public, 14 people who had had a stroke, and 10 carers or partners.

Main outcome measures Views on risk factors, symptoms, treatment, information resources, and prevention.

Results All groups reported similar knowledge of risk factors. People generally mentioned stress, diet, high blood pressure, age, and smoking as causes of stroke. Participants in the community group gave little attention to symptoms. Some participants who had had a stroke did not initially identify their experience as stroke because the symptoms were not the same as those they had read about. There were mixed feelings about the extent of involvement in management decisions during hospital admission. Some felt sufficiently involved, some wanted to be more involved, and others felt incapable of being actively involved.

Conclusions Symptoms of stroke are not easy to recognise because they vary so much. Presentation of information about stroke by hospital and community health services should be improved. Simple and understandable educational materials should be developed and their effectiveness monitored.
\end{abstract}

\section{Introduction}

Studies of acute intervention for stroke have shown that outcome is more favourable if the symptoms are recognised early. However, most people do not seek timely medical attention. ${ }^{1-4}$ Many factors contribute to delays in seeking medical treatment for acute stroke, but one that should be remediable is public lack of knowledge about symptoms, which often results in delay in seeking medical care. ${ }^{5}$

Our previous study on public perception of warning signs, symptoms, and treatment of stroke in an urban area of Australia showed that only $73 \%$ of respondents identified the brain as the organ affected by stroke. ${ }^{6}$ When asked how they would respond to the occurrence of a stroke, $90 \%$ of respondents said they would call an ambulance or visit a hospital emergency department. However, when asked about how they would respond to various symptoms, without reference to stroke, only $23-42 \%$ indicated that they would respond in either of these recommended ways. Over half of respondents did not know of any of the existing organisations that provided information about stroke or support to patients and their families.

We have previously shown there is a lack of information available to people in the community. ${ }^{7}$ This deficiency continues despite evidence showing that better knowledge is associated with early presentation in hospital emergency departments. ${ }^{5}$ The benefits of hospital based education and counselling that deals with the emotional and social concerns of people with a stroke and of their carers have been described..$^{910}$ Effective community education programmes are vital to increase public awareness of stroke.

We carried out a qualitative study to obtain insight into people's thoughts on stroke, including risk factors, symptoms, treatment, information resources, and prevention; to inform the development of an educational strategy for the early recognition of symptoms and for appropriate responses to these in the community; and to inform the development of an educational programme for people who have had a stroke.

\section{Method}

Design of study -We conducted focused discussions with groups of people who had had a stroke and their carers (in two groups) and with members of the general public (in two groups). The group discussions took place in a non-clinical setting in hospital. The study was approved by the Newcastle University and Hunter Area research ethics committees.

Sample-We selected people who had had a stroke from the heart and stroke register in Hunter Area Health Authority. ${ }^{11}$ All of them were living in the community and had agreed to be contacted for participation in further studies. The register sent 87 information letters and consent forms to people who had had a stroke between July 1999 and July 2000. Of those, 56 letters were returned, and 27 people agreed to participate. We contacted each person to organise a date for group discussions. Fourteen out of 27 people
Centre For Clinical
Epidemiology and
Biostatistics, Faculty
of Medicine and
Health Sciences,
University of
Newcastle, New
South Wales 2308,
Australia
Sung Sug Yoon
nurse
Julie Byles
associate professor
Correspondence to:
S Yoon
sungsyoon@
yahoo.com
bmj.com 2002;324:1065 
who had had a stroke and 10 carers attended. We recruited 11 people who had not had a stroke from the local area using a snowballing technique. This method entails identifying initial participants in the study group who go on to recommend other people for recruitment. Each of the subsequently interviewed participants is asked for further recommendation. ${ }^{12}{ }^{13}$ The method is also useful in the generation of hypotheses and to obtain an idea of the range of responses on ideas that people have. ${ }^{14}$

Discussion guide and procedure - We developed a discussion guide on the basis of findings of previous studies. ${ }^{6716-21}$ Six items dealt with knowledge and perceptions of risk factors, symptoms, treatment, information resources, and reaction to symptoms. Participants also completed questionnaires that were collected anonymously. The questionnaires included items on age, sex, marital status, country of origin, education, income, and self reported risk factors (high blood pressure, angina, heart attack, previous stroke, diabetes, high cholesterol concentration, smoking, and family history of stroke). Each discussion group was moderated by the same two researchers, who ensured that each group fully discussed each item on the agenda and that all respondents had sufficient opportunity to air their views. A moderator introduced the topic and assisted the participants to discuss it, encouraging interaction and guiding the conversation. Discussions lasted 60-90 minutes, with an additional 30 minutes for refreshments and informal conversation. Every session was audiotaped, with the written consent of each participant, and transcribed verbatim. At the end, information was provided regarding support services available for people with stroke and their carers. Participants were offered the opportunity to review the transcripts and the final analysis.

\section{Analysis}

We developed higher codes from the data, including definition, risk factors, symptoms, treatment, reaction, differences, and information. We read and re-read the transcripts and notes and organised data into initial codes, then into higher codes that provided insight into identified themes. For example, one participant mentioned "It is very repeated and everything you pick up is telling you the same things" to one question in the discussion guide ("has anyone ever seen a pamphlet or poster or TV commercial on stroke? How did you feel about the information?"). This goes to initial code "presentprg", which represented data for evaluation of present programmes then into higher codes on "information" which included initial codes of "presentprg" and "preferprg" (prefer programmes for stroke information).

We identified and discussed a hierarchical scheme of specific themes, issues, and problems that emerged from the data. We used the computer package Ethnograph 5.0 to analyse data more conveniently and effectively. ${ }^{22}$

\section{Results}

Thirty five people attended meetings: 11 from the general public ( $85 \%$ of those invited), 14 of the 27 people who had had a stroke and agreed to participate ( $16 \%$ of those invited), and 10 carers or partners. The table shows demographic characteristics for patients and the general public.

\section{Perceptions of stroke}

Participants in both groups described stroke as a clot or a bleed. These participants believed that a blood clot went into the brain and blocked the blood circulation, which caused the affected part of the brain to become inactive. This resulted in the body being affected in one or more ways. Participants who had experienced a stroke were more likely to speak about stroke in their own idiom rather than using terminology from text books or available educational information-for example, "Headness, just headness-you know it was not headache at all-it's like something is going on in my brain-also dizziness."

Participants were asked questions about the possibility of having a stroke. Most regarded the thought of any illness as an unnecessary additional worry. Indeed, they avoided thinking about any illness or other adverse events. There was a greater focus on the risk of heart attack or cancer than on the risk of having a stroke. Before they had a stroke most participants in the patient group had never thought about their lifetime chance of having a stroke and some knew nothing about stroke.

\section{Risk factors and symptoms of stroke}

All groups reported similar knowledge of risk factors for stroke. People generally mentioned stress, diet, high blood pressure, age, smoking, and genetics as causes of stroke. Few people believed that stroke can occur without any cause or without the presence of risk factors. Some people particularly emphasised stress and diet: "I always imagined that stress and frustration affected blood pressure, which sent it sky high, and then the blood pressure brought on the stroke"; "We are getting much fatter, because we're eating so much more. Because everywhere you go there is so much you can eat and it is so easy." With regard to prevention, people pointed to community education, change of life style, and school or institution programmes. Descriptions of symptoms by people who had had a stroke (box 1) differed from descriptions by members of the general public group, which tended towards terminology found in textbooks or in National Stroke Association publications. ${ }^{23}$

\section{Response to stroke symptoms}

Box 2 shows how people in the general public group said they would respond to symptoms of stroke and

Characteristics of participants in group discussions of awareness of stroke

\begin{tabular}{lcc} 
& $\begin{array}{c}\text { Community groups } \\
(\mathbf{n}=\mathbf{1 1})\end{array}$ & $\begin{array}{c}\text { Stroke groups* } \\
(\mathbf{n}=\mathbf{1 4})\end{array}$ \\
\hline Mean (SD) age (years) & $64.0(9.3)$ & $70.1(5.6)$ \\
\hline Men & 4 & 7 \\
\hline Women & 7 & 7 \\
\hline Country of origin: & 11 & 13 \\
\hline Australia & 0 & 1 \\
\hline Overseas & 2 & 10 \\
\hline High blood pressure & 8 & 3 \\
\hline High cholesterol & 0 & 2 \\
\hline Heart disease & 0 & 2 \\
\hline History of stroke & 2 & 6 \\
\hline Family history of stroke & 2 & 2 \\
\hline Current smoking & &
\end{tabular}




\section{Box 1: Key comments-recognition of} symptoms

\section{General public}

Had a stroke, people just think of paralysis Swallowing and different things happen Personality changed completely

Loss of vision for no reason

Lost control of her bladder

Tremors in your hand

Pain or something like that, maybe dizziness

Tingling sensations

Memory loss

Headaches

Blurred vision

\section{Stroke groups}

Emotional incontinence; easily laughing and crying Speech sounds like a bird or as if drunk

Headache

Feel funny, feel heavy, strange feeling on my face Right side falling

Face looking dreadful

Had taste like pine taste

Light headedness

No headache just bang and crying

Tongue was a bit funny for a while

The left side of my face, especially my mouth area, felt strange and tingly

Getting a little strange while I was walking around like a drunk

I lost the use of my left arm and collapsed

I had no feeling in my right arm, my face went a bit funny

Balance and double vision

how patients who had had a stroke did respond to their symptoms. Participants in the general public groups placed little importance on the symptoms. In reaction to the symptoms of numbness, tingling sensation, and weakness or paralysis of one side of the body some of them said they would lie down and take couple of paracetamol. But if they experienced difficulties in speaking (which they perceived as definitely abnormal), they would seek urgent medical attention.

Many in the stroke groups initially did not take their symptoms seriously and had waited for symptoms to abate. Most did not realise that the symptoms were related to stroke: "... not thinking it was a stroke, I had no idea," "when I had the stroke, I didn't know what it was, I never had a clue," "I didn't actually know what it was because I have never been sick." Some did not identify their experience as stroke because the symptoms did not present as expected. Both groups thought that they would not receive any medical attention for minor symptoms such as headache and dizziness.

\section{Treatment and expectation of treatment}

Participants in both groups referred to rehabilitation as including physiotherapy, speech therapy, and occupational therapy. Some people described a drug for dissolving clots in the blood vessels and procedures for removing a clot from the artery, such as endarterectomy. Most of those who had had a stroke were aware of their own drug treatment and were concerned about the side effect of drugs. Box 3 shows their expectations of treatment after admission to hospital. A serious difficulty encountered during admission was

\section{Box 3: Expectations of treatment in stroke} groups

Important to know information like what is going on Don't know what are the expected symptoms-what will happen

Hospital did not give any treatments, just asked, "where were you, what day is it?" Sick of MMSE [mini-mental state examination]

I felt as though my treatment was passive treatment, I think I was resting more in hospital than I may have been resting at home

Hospital, not seen as a place to get better or rehabilitate

Did not explain what is going on at the hospital One day they [doctor] will have a stroke and know what it's like

They are the experts and you go along with what the experts think is best for you

I don't think I have been involved. I think I need more explanation to be honest

The doctor said to me now you have had a stroke and you are going home and this is what you ought to do Something physically, something mentally

that healthcare providers did not give satisfactory information about aspects of the treatment. There were mixed feelings about the extent of involvement in management decisions. Some felt sufficiently involved, some wanted to be more involved, and others felt incapable of being actively involved.

\section{Box 2: Response to symptoms}

General public

There is nothing you can do, you just ring the ambulance

If you have enough stress you might get a headache but you certainly don't get palpitations or shortness of breath

We were not really brought up to go to the doctors very much in the country and doctors weren't as readily available

Severity is the most important thing. If it is not within your experience, it is something that you worry about Your experience tells you that most times, almost every time, it has gone away in time

\section{Stroke groups}

Did not worry much about it and took medication for migraine and wait

Thought symptoms will be better tomorrow

Time was late so just went to bed thinking it will be better after sleep

Thought it was something else like food poisoning I wondered what have I eaten or am I having a stroke? I will see doctor tomorrow, I think

It was the weekend and I don't think to see a doctor about nothing because they have the weekend off, and I'd rather see them stay home, so I go on Monday I only think vomiting at home would have been the flu and you don't worry about it

Just because you have a severe headache you don't assume you are having a stroke, just because your face is tingling (because when you have a history of an allergy that is similar) you don't assume you are having a stroke

I mean I'd know instantly if somebody's face was drooping or drooling but in this case there is no meaning 
Differences between stroke and heart attack

There was some confusion in the groups between heart attack and stroke. Some participants in stroke groups identified "pain" in the chest as the distinguishing sign of heart attack. Other participants said that the only difference was whether the blood clot goes to the heart or to the brain. Some people in the general public group thought stroke was more serious than heart attack: "You'd much rather have a heart attack than a stroke because, a heart attack you get over it, and you get to do things right. A stroke needs such a long rehab and time," "The stroke, there is more than that, the heart seems to-clear out the arteries, and will start to work again, because it's only affecting the circulation, but the brain-seems that doesn't come back." Recognising symptoms of stroke in the community is seen as more difficult than recognising heart attack because stroke symptoms are much more heterogeneous. For example, "Your heart attack is sort of easier because of the chest pain, difficulty breathing, pain in your arm that sort of thing... Stroke, because it depends, what the function of that part of the brain is."

\section{Information resources}

Participants in the general public groups knew little about stroke organisations or available educational materials such as pamphlets, booklets, and leaflets. Box 4 shows participants' preferences regarding educational programmes and their evaluation of current education programmes in the area. Most people expected to receive information from their general practitioner or from community education. They preferred simple and understandable messages rather than repetitive (confusing) messages.

\section{Discussion}

This qualitative study shows that people in the community have similar understandings about the description of stroke and the possibility of having a stroke regardless of whether or not they have had a stroke. Participants expressed the view that recognising symptoms is not easy. Many patients who had had a stroke did not initially take their symptoms seriously because the symptoms did not fit the typical pattern presented in information they had received. The stroke groups emphasised that more information needs to come from hospitals as well as from community health services. Both groups in this study wanted education programmes to contain simple and understandable information.

\section{Perception of stroke}

Participants were disinclined to accept that they were ill or at risk of any kind of illness. They did not want to accept illness as part of their life. We have previously shown that people are more likely to say they have a low lifetime risk of stroke. ${ }^{7}$ These attitudes towards illness may counteract attempts to increase awareness of stroke in the community. Educational strategies may need to focus on the positive benefits of healthy lifestyles rather than on the negative results of risky lifestyles.

Most participants envisaged stroke as more serious than heart attack. However, because stroke symptoms present in various ways they are not easy to recognise. People who had had a stroke said that they had confused their symptoms with those of migraine, food poisoning, and Ménière's disease. This kind of confusion may be one cause for delay in presentation to hospital. Studies in people with myocardial infarction showed that presentation with atypical symptoms may be responsible for increased delays in many patients. ${ }^{24}{ }^{25}$ Dracup et al found that people who thought they only had heartburn or indigestion significantly delayed seeing a doctor. ${ }^{26}$

\section{Response to symptoms}

The lack of knowledge about stroke was one of the reasons for delay in early presentation at hospital. Previously our telephone survey revealed that most of the respondents $(90 \%)$ would consider calling an ambulance or visiting a hospital casualty or emergency department if they thought that they were having a stroke. However, when asked how they would respond to particular symptoms, without reference to stroke, less than half and as few as 3\% indicated that they would respond in this way. ${ }^{6}$ Other studies showed similar reasons for delay in hospital presentation. ${ }^{87}$ Most patients in our study waited until the next morning or after the weekend because they believed that the

\section{Box 4: Information resources \\ General public \\ Preferred programmes \\ Tell the people, educate the people with straight out information and make it really simple to understand Just educate the community as to what a stroke is because a lot of them wouldn't know the possible symptoms of a stroke \\ A TV commercial, because it shows that recovery is very achievable At high school}

Evaluation of present programmes

If you're going to worry people that much, if you say "if you've slight headache, ring for an ambulance, if you've got numbness in your arm," you're going to have so many calls, so many false alarms that it's going to probably prove to be a bigger headache than non-education

\section{Stroke groups}

Preferred programmes

TV is the best

Family members or friends

Self help groups

Being constructive rather than reading the same thing over and over, which doesn't sink in, like always, I have a bit of bother, I read a lot but sometimes I get through part of the chapter and think what did I read, it is just not getting through, so printed it might not get through

If GPs were to hand down little leaflets, maybe one day you will have a stroke, this is what you need to look out for, maybe if these little leaflets were handed out to us, like us people who have high blood pressure or whatever, diabetes, high cholesterol, whatever our problems are

Evaluation of present programmes

Don't want to go [to stroke support group] because it makes me depressed and helpless, some people can't go because of severe disability, need to have a group to share information and encourage

It is very repeated and everything you pick up is telling you the same things 


\section{What is already known on this topic}

Increasing the speed of presentation to hospital after the onset of stroke depends on the level of knowledge of stroke in the general population

Among stroke patients and the general public the knowledge of stroke is poor

\section{What this study adds}

Focus group discussion showed that recognition of stroke was not easy for the general public because symptoms present in various ways

None of the available written information about stroke successfully conveyed the importance of early presentation to hospital for anyone experiencing warning signs or symptoms

symptoms would go away soon. To target populations at risk it is important to emphasise knowledge of symptoms and the appropriate response, but it is also to understand the role of defence and coping mechanisms.

Participants in both groups believed that they would not get any medical attention for symptoms that they thought were not typical or symptoms of minor stroke. Although the general public have enough knowledge to recognise symptoms of stroke, preoccupation about medical attention may contribute to delayed presentation. One prospective observational study showed that only a third of patients with myocardial infarction but without chest pain were correctly diagnosed and mortality in hospital was 23\% compared with $9 \%$ among patients with chest pain. Patients without chest pain also presented later at hospital compared with those with chest pain. ${ }^{28}$ Change in the attitudes of healthcare providers may encourage people who are not sure about their symptoms to present earlier.

\section{Information resources}

Patients in our study reported that they had not received enough information from healthcare providers during their stay in hospital. An intensive educational programme in hospital may not be effective in the early stage of the relationship between patients, carers, and doctors because stroke, especially in the acute stage, excessively burdens people with other concerns (for example, losing a job and financial and other fears). Previous studies have shown that the quality of relationship between the doctor and patient influences the patient's satisfaction and compliance with treatment. ${ }^{29}{ }^{30}$ In the current study information about stroke from healthcare providers may not have been effectively transmitted to patients and carers or not retained. Discharge plans could incorporate provision of information about stroke prevention by a community stroke service.

Participants in our study recommended community education about recognising stroke and about appropriate responses by people who experience stroke. Some such educational programmes have been evaluated in other countries. ${ }^{13} 17$ However, planners may need to evaluate the cost effectiveness of each educational project and consider its long term effects. As our participants emphasised, educational programmes (including printed information, visual and audio programmes, and community stroke service programmes) need to use simple and understandable information and focus on the population as a whole as well as on people at high risk.

Contributors: SSY wrote the first draft of the manuscript and contributed to design, recruitment of participants, data collection, and analysis and is guarantor. JB contributed to design, data collection, and analysis and read and discussed the manuscript.

Funding: None.

Competing interest: None declared.

1 Del Zoppo GJ, Higashida RT, Furlan AJ, Pessin MS, Rowley HA, Gent M. PROACT: a phase II randomized trial of recombinant pro-urokinase by direct arteria delivery in acute middle cerebral artery stroke. Stroke 1998;29:4-11.

2 Donnan GA, Davis SM, Chambers BR, Gates PC, Hankey GJ, McNeil JJ, et al. Streptokinase for acute ischemic stroke with relationship to time of administration. JAMA 1996;276:961-6.

3 Hacke W, Kaste M, Fieschi C, Toni D, Lesaffre E, Kummer RV, et al. Intravenous thrombolysis with recombinant tissue plasminogen activator for acute hemispheric stroke: the European cooperative acute stroke study (ECASS). JAMA 1995;274:1017-25.

4 National Institute of Neurological Disorders and Stroke rt-PA Stroke Study Group. Tissue plasminogen activator for acute ischemic stroke. N Engl J Med 1995;333:1581-7.

5 Alberts MJ. tPA in acute ischemic stroke: United States experience and issues for the future. Neurology 1998;51(suppl 3):S53-5.

6 Sug Yoon S, Heller RF, Wiggers J, Levi C, Fitzgerald PE. Knowledge of stroke risk factors, warning symptoms, and treatment among an Australian urban population. Stroke 2001;32:1926-30.

7 Yoon SS, Heller R, Levi C, Wiggers J. Knowledge and perception about stroke among an Australian urban population. BioMed Central Pub Health 2002;1:14.

8 Ferro JM, Melo TP, Oliveira V, Crespo M, Canhao P, Pinto AN. An analysis of the admission delay of acute stroke. Cerebrovasc Dis 1994;4:72-5.

9 Wood VA, Hewer RL. The prevention and management of stroke. J Pub lic Health Med 1996;18:423-31.

10 Evans RL, Connis RT, Bishop DS, Hendricks RD, Haselkorn JK. Stroke: a family dilemma. Disabil Rehabil 1994;16:110-8.

11 Heart and Stroke Health Outcomes Council. Heart and stroke register. Newcastle, NSW: Heart and Stroke Health Outcomes Council, 2001.

12 Bernard H. Research methods in anthropology. Beverly Hills, CA: Sage, 1988

13 Babbie E. The practice of social research. 6th ed. Belmont, California: Wadsworth, 1992.

14 Black JA, Champion D. Methods and issues in social research. Chichester: John Wiley, 1976

15 Hendricks VM, Blanken P, Adriaans N. Snowball sampling: a pilot study on cocaine use. Rotterdam: IVO, 1992

16 Alberts MJ, Perry A, Dawson DV, Bertels B. Effects of public and professional education on reducing the delay in presentation and referral of stroke patients. Stroke 1992;23:352-6.

17 Barsan WG, Brott TG, Broderick JP, Haley EC, Levy DE, Marler JR. Time of hospital presentation in patients with acute stroke. Arch Intern Med 1993;153:2558-61.

18 Harper GD, Haigh RA, Potter JF, Castleden CM. Factors delaying hospital admission after stroke in Leicestershire. Stroke 1992;23:835-8

19 Hartford M, Karlson BW, Sjolin M, Holmberg S, Herlitz J. Symptoms, thoughts, and environmental factors in suspected acute myocardial infarction. Heart Lung 1993;22:64-70.

20 Stern EB, Berman ME, Thomas JJ, Klassen AC. Community education for stroke awareness an efficacy study. Stroke 1999:30:720-3.

21 Wester P, Radberg J, Lundgren B, Peltonen M, and for the Seek-MedicalAttention-in Time Study Group. Factors associated with delayed admission to hospital and in-hospital delays in acute stroke and TIA: a prospective, multicenter study. Stroke 1999;30:40-8.

22 Scolari. Ethnograph v5.0. London: Sage, 1999.

23 National Stroke Association. Stroke risk factors and their impact. www.stroke.org/stroke_risk.cfm (accessed 24 April 2002).

24 Moser DK, Dracup K. Gender differences in treatment-seeking delay in acute myocardial infarction. Prog Cardiovasc Nurs 1993;8:6-12.

25 Schwarz B, Schoberberger R, Rieder A, Kunze M. Factors delaying treatment of acute myocardial infarction. Eur Heart J 1994;15:1595-8.

26 Dracup K, McKinley SM, Moser DK. Australian patients' delay in response to heart attack symptoms. Med J Aust 1997;166:233-6.

27 Feldmann E, Gordon N, Brooks JM, Brass LM, Fayad PB, Sawaya KL, et al. Factors associated with early presentation of acute stroke. Stroke 1993;24:1805-10

28 Canto JG, Shlipak MG, Rogers WJ, Malmgren JA, Frederick PD, Lambrew $\mathrm{CT}$, et al. Prevalence, clinical characteristics, and mortality among patients with myocardial infarction presenting without chest pain. JAMA 2000;283:3223-9.

29 Hill MN. Strategies for patient education. Clin Exp Hyperten 1989;11:1187-201. 
Papers

30 Legato MJ, Padus E, Slaughter E. Women's perceptions of their general health, with special reference to their risk of coronary artery disease:

(Accepted 22 November 2001) results of a national telephone survey.J Womens Health 1997;6:189-98. 